\title{
RECOGNIZING SOME GEOMETRICAL OBJECTS FROM A DISCRETE SET OF POLYGONS
}

\author{
NGO HOANG HUY - NGO QUOC TAO ${ }^{(1)}$
}

\begin{abstract}
This paper presents a post - processing method for extracting polygons after vectorization processing. These polygons are used for a Spatial Data Type of Geographic Information Systems or for recognizing primitive objects included in technical drawings. We propose the semi automatic searching method for extrating from a set of polylines to a polygon surrounding a given point. This method is based on the depth searching strategy. Then we give a measure fitting polygons with primitive geometrical objects. In fact, the paper makes a deal with the applying of global statistical and geometrical invariants for reconizing planar objects.
\end{abstract}

\section{NHẬN DẠNG MộT SỐ ĐỐI TƯợNG HİNH HỌC TỪ CÁC TẬP ĐƯờNG GẤP KHÚC RỜI RẠC}

\author{
NGÔ HUY HOÀNG - NGÔ QUỐC TAOO(1)
}

Tóm tắt. Bài báo trình bày phương pháp trích chọn các đa giác sau quá trình véc tơ hoá. Có thể dùng các đa giác này cho kiểu dữ liệu không gian trong các hệ thống thông tin địa lý hoặc nhận ra đối tượng cơ bản trong các bản vẽ kỹ thuật. Chúng tôi thiết lập thủ tục tìm kiếm bán tự động một đa giác bao quanh một điểm cho trước từ tập các đường gấp khúc. Phương pháp này dựa theo chiến thuật tìm kiếm theo chiều sâu. Sau đó chúng tôi đưa ra độ đo xấp xỉ đa giác với các dạng hình học chuẩn. Về mặt thực hành bài báo đã đề cập đến cách ứng cung bất biến thống kê và hình học toàn cục đối với việc nhận dạng đối phẳng.

\section{GIỚI THIÊU}

Quá trình xây dựng hệ bản đồ, các hệ trợ giúp nhập liệu tự động tỏ rõ tính năng ưu việt trong vấn đề về thời gian, song cho đế $i$ này chưa có một thuật toán hữu hiệu nào đảm bảo yêu cầu về tốc độ, tính chất tôpô (điểm khớp), nhận dạng tự động các dạng hình học cơ bản như: đường tròn, ellipse, đa giác v. v... (xem hình [1]). Điều trở ngại này xuất hiện do tính chất ngẫu nhiên của việc lựa chọn hướng tìm kiếm xuất phát từ một điểm của các thuật toán véc tơ hoá. Vi thế quá trình hậu xử lý khi véc tơ hoá là cần thiết để "dán và nắn lại" các đường gấp khúc "rời nhau" thành các dạng hình học cơ sở.

Phần còn lại của bài báo như sau:

Mục 2 đưa ra một số định nghĩa hình thức. Mục 3 trình bày phương pháp trích chọn một đa giác dựa vào một điểm gieo. Mục 4 đề xuất một phương pháp xấp xỉ đa giác bởi các hình cơ sở như tam giác, hình chữ nhật, hình vuông, hình ellipse, hình tròn v. v... 


\section{CẤU TRÚC CƠ BẢN CỦA DŨ LIÊUU VÀO}

Đinh nghĩa 1: Ta có thể mô tả theo cấu trúc cây của các thành phần sau khi véc tơ hoá (xem $[2,3]$ ) như sau:

- Điểm là một cặp số thực $(\mathrm{x}, \mathrm{y})$. Khoảng cách giữa hai điểm $\left(\mathrm{x}_{1}, \mathrm{y}_{1}\right)$ và $\left(\mathrm{x}_{2}, \mathrm{y}_{2}\right)$ được xác đinh như sau:

$$
d\left(\left(x_{1}, y_{1}\right),\left(x_{2}, y_{2}\right)\right)=\max \left(\left|\mathrm{x}_{1}-\mathrm{x}_{2}\right|,\left|\mathrm{y}_{1}-\mathrm{y}_{2}\right|\right)
$$

- Đoạn thẳng là một tập điểm hai phần tử $\left\{\mathrm{p}_{1}, \mathrm{p}_{2}\right\}$.

- Đường gấp khúc là dãy các điểm $\left.<\mathrm{p}_{1}, \mathrm{p}_{2}, \ldots, \mathrm{p}_{\mathrm{n}}\right\rangle$, khi đó $\mathrm{p}_{\mathrm{l}}$ và $\mathrm{p}_{\mathrm{n}}$ được gọi là các điểm cuối của đường gấp khúc.

Tập các đường gấp khúc thoả mãn hai tính chất sau:

i) Không tụ c'ắt

ii) Giao c'ủa hai đường gấp khúc là duy nhất tạo thành c'ác điểm chụm (xem [1]).

Thông thường một đa giác được hiểu như là một đường gấp khúc "khép kín" (sai khác một dung sai). Do đặc thù dữ liệu vào sau quá trình véc tơ hoá chúng ta cần phải mở rộng định nghĩa của một đa giác như sau:

Đinh nghĩa 2: Cho trước một dung sai $\theta$, hai đường gấp khúc $\mathrm{Pl}$ và $\mathrm{Pl}$ ' gọi là khớp nhau nếu khoảng cách hai điểm cuối nào đó của $\mathrm{Pl}$ và $\mathrm{Pl}$ ' nhỏ hơn dung sai $\theta$.

Đinh nghĩa 3: Cho trước dung sai $\theta$, dãy các đường gấp khúc $\left.<\mathbf{P I}_{1}, \ldots, \mathbf{P l}_{\mathrm{n}}\right), \mathrm{n} \geq 2$ được gọi là đa giác nếu nó thoả mãn các điều kiện sau:

$$
\begin{aligned}
& \text { i) } P l_{n} \equiv P l_{l} \text {. } \\
& \text { ii) } P l_{i} \text { khóp với } P l_{i+1} \\
& \text { iii) } \forall i ! \exists j \neq i+1 ; P l_{i} \text { khớp với } P l_{j}
\end{aligned}
$$

\section{THUÂT TOÁN TİM KIẾM VÀ XẤP XỈ ĐA GIÁC}

Quá trình tìm kiếm các đa giác được hình thức hoá như sau:

Bài toán: (Bài toán tìm kiếm tự động tất cả các đa giác )

Dũ liêu vào: Cho trước dung sai $\theta$, hàm khoảng cách $\mathrm{d}$ và tập hợp rời rạc các đường gấp khúc $\{\mathrm{Pl}\}$ thoả mãn các tính chất i) và ii).

Kết quả: Tập hợp các đa giác $<\mathrm{Pl}_{1}, \mathrm{Pl}_{2}, \ldots \mathrm{Pl}_{\mathrm{n}}$ ).

Bài toán này phức tạp khi đưa ra các đa giác bao nhau và có chung cạnh, tốc độ bị ảnh hưởng khi thực hiện tìm kiếm vét cạn. Để xác định một đa giác duy nhất khi tìm kiếm chúng tôi hạn chế bài toàn này bằng cách chỉ tìm đa giác cho bao quanh một điểm cho trước, được phát biểu như sau:

\section{Bài toán 1: (Bài toán tìm kiếm)}

Dũ liêu vào: Cho trước điểm $\mathrm{M}\left(\mathrm{x}_{0}, \mathrm{y}_{\mathrm{o}}\right)$, dung sai $\theta$, hàm khoảng cách $\mathrm{d}$ và tập hợp rời rạc các đường gấp khúc $\{\mathbf{P I}\}$ thoả mãn các tính chất iv) của định nghĩa 1 .

Kết quả: đa giác $\left.<\mathrm{Pl}_{1}, \mathrm{Pl}_{2}, \ldots \mathrm{Pl}_{\mathrm{n}}\right\rangle$.

Bài toán 2: Nhận biết một đa giác theo các dạng hình cơ sở.

Tìm đa giác. 
Rõ ràng việc tìm kiếm một dãy "khớp" các đường gấp khúc là phụ thuộc vào việc chọn thành phần $\mathrm{Pl}_{1}$ đầu tiên, các thành phần $\mathrm{PI}_{\mathrm{i}+1}$ có thể được đưa ra nhờ việc xây dựng quá trình tìm kiếm theo chiều sâu các đường gấp khúc khớp với $\mathbf{P I}_{\mathrm{i}}$, để việc tìm kiếm được nhanh chóng, chúng ta cần một kiểm nghiệm đơn giản để chọn được thành phần $\mathbf{P l}_{\mathbf{i}+1}$ có khả năng dẫn đến lời giải "tốt hơn" so với các đường gấp khúc khác kề với nó. Chúng ta sẽ dựa vào điểm $\mathrm{M}\left(\mathrm{x}_{0}, \mathrm{y}_{\mathrm{o}}\right)$ để điều khiển quá trình tìm kiếm.

Ta định nghĩa góc giữa hai đường gấp khúc "khớp nhau" như sau:

Đinh nghĩa 4: Giả sử $\mathbf{P}$ và $Q$ là hai đường gấp khúc khớp nhau tại hai điểm cuối $\mathrm{A}$ và $\mathrm{B}$

tương ứng. Ta gọi góc giữa $\mathbf{P}$ và $\mathbf{Q}$ là $(\mathbf{P}, \mathbf{Q})=\left(\mathrm{A}^{\prime} \mathrm{A}, \mathrm{B}^{\prime} \mathrm{B}\right)$, ở đây $\mathrm{A}^{\prime} \mathrm{A}, \mathrm{B}^{\prime} \mathrm{B}$ là hai cạnh của $\mathbf{P}$ và $\mathbf{Q}$ tương ứng.

Tiêu chuẩn rẽ nhánh cho chiến thuật tìm kiếm chiều sâu:

Từ $\mathbf{P l}_{i}$ chọn $\mathbf{P l i}+1 \in \mathbf{L i s t M a t c h}(\mathbf{P l i})$ sao cho:

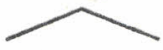

(Pli, Pli +1$) \rightarrow \min$

Ở đây kí hiệu ListMatch $\left(\mathrm{Pl}_{\mathrm{i}}\right)$ là danh sách các đường gấp khúc khớp với $\mathrm{Pl}_{\mathrm{i}}$.

Tính chất của đa giác kết quả là:

i) Chúa diểm $M\left(x_{0}, y_{0}\right)$.

ii) Có it nhất một đoạn thäng nối $M\left(x_{0}, y_{0}\right)$ với các đỉnh của đa giác mà không cắt bất kỳ đường gấp khúc nào của tập dữ liệu vào.

Với phương pháp tìm kiếm theo chiều sâu, chúng ta sẽ tìm đa giác bằng cách duyệt trên các đường gấp khúc như sau:

Bước O:

Duyệt trên tập dữ liệu vào, nếu $\mathrm{M}$ gần một đỉnh của đường gấp khúc nào đó một khoảng cách nhỏ hơn $\theta$ thì thay thế $M$ bởi đỉnh này.

\section{Buóc l:}

Tìm đường gấp khúc gần nhất ở bên trái $\mathrm{M}\left(\mathrm{x}_{0}, \mathrm{y}_{0}\right)$ và kí hiệu là $\mathrm{Pl}_{1}$.

Nếu $<\mathrm{Pl}_{1}, \mathrm{Pl}_{1}>$ là đa giác "à thoả mãn tính chất iii) và iv) thì chuyển tới bước 3 .

Bước 2:

Duyệt các đường gấp khúc chưa bị đánh dấu, nếu cso.

Tìm $\mathrm{Pl}_{i+1}$ trong ListMatch $\left(\mathrm{Pl}_{i}\right)$ thoả mãn điều kiện (*).

Nếu $\left\{\mathrm{Pl}_{1} \ldots \mathrm{Pl}_{\mathrm{i}}, \mathrm{Pl}_{\mathrm{i}+1}\right\}$ thra mãn tính chất i) và ii) thì nhảy tới bước 3 , trái lại đánh dấu $\mathrm{Pl}_{\mathrm{i}+1}$ và quay lại bước 2 .

Trái lại, kết thúc và thông báo không tồn tại đa giác chứa điểm $\mathrm{M}$.

\section{Bước 3:}

Kết thúc và trả lại đa giác $\left\langle\mathrm{Pl}_{1}, \mathrm{Pl}_{2}, \ldots \mathrm{Pl}_{n}\right\rangle$.

Trong cài đặt thủ tục tìm kiếm trên chúng tôi đã sử dụng thü tục kiểm tra xem một điểm có nằm trong một đa giác cho trước hay không bằng cách dựa vào tính chẵn lẻ của số giao điểm (xem [2]). Thủ tục tìm kiếm có thể phát hiện được các đa giác có dạng như hình $1 \mathrm{a}$ ) và $1 \mathrm{~b}$ ). Trong Hình $1 \mathrm{~b}$ ) nếu lấy điểm $\mathrm{M}$ là điểm ngã tư $\{9,10,11,12\}$ thì ta tìm được một đa giác duy nhất $<2,3,5,7\rangle$. 


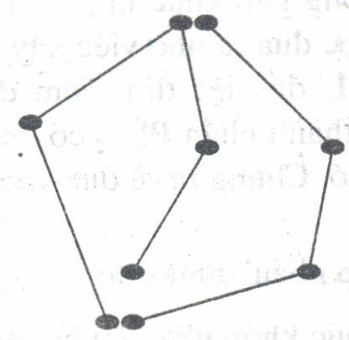

(a)

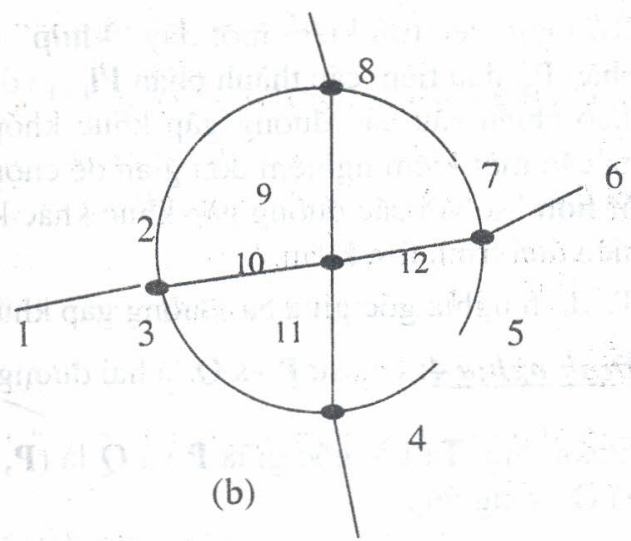

(b)

Hình 1.a) và b) biểu diễn tập các đường gấp khúc.

\section{NHẬN DẬG ĐA GIÁC.}

Một đa giác có thể có hình dạng tựa như một hình cơ sở, có thể có nhiều cách tiếp cận xấp xỉ khác nhau. Cách xấp xỉ dựa trên các đặc trưng cơ bản sau:

- Đặc trưng toàn cục: Các mômen thống kê, số đo hình học như chu vi, diện tích, tập tối ưu các hình chữ nhật phủ hay nội tiếp đa giác v. v.

- Đặc trưng địa phương: Các số đo đặc trưng của đường cong như góc, điểm lồi, lõm, uốn, cực trị. v. v...

Dựa trên các tiếp cận được đưa ra trong các tài liệu $[5,6,7,8]$ chúng tôi lựa chọn tiêp cận với đặc trưng toàn cục và đưa ra sơ đồ xấp xỉ theo các bất biến đồng dạng và bất biến aphin. Việc xấp xỉ tỏ ra rất có hiệu quả đối với một số hình phẳng đặc biệt như tam giác, đường tròn, hình chữ nhật, hình vuông, hình ellipse, hình tròn và một đa giác mẩu.

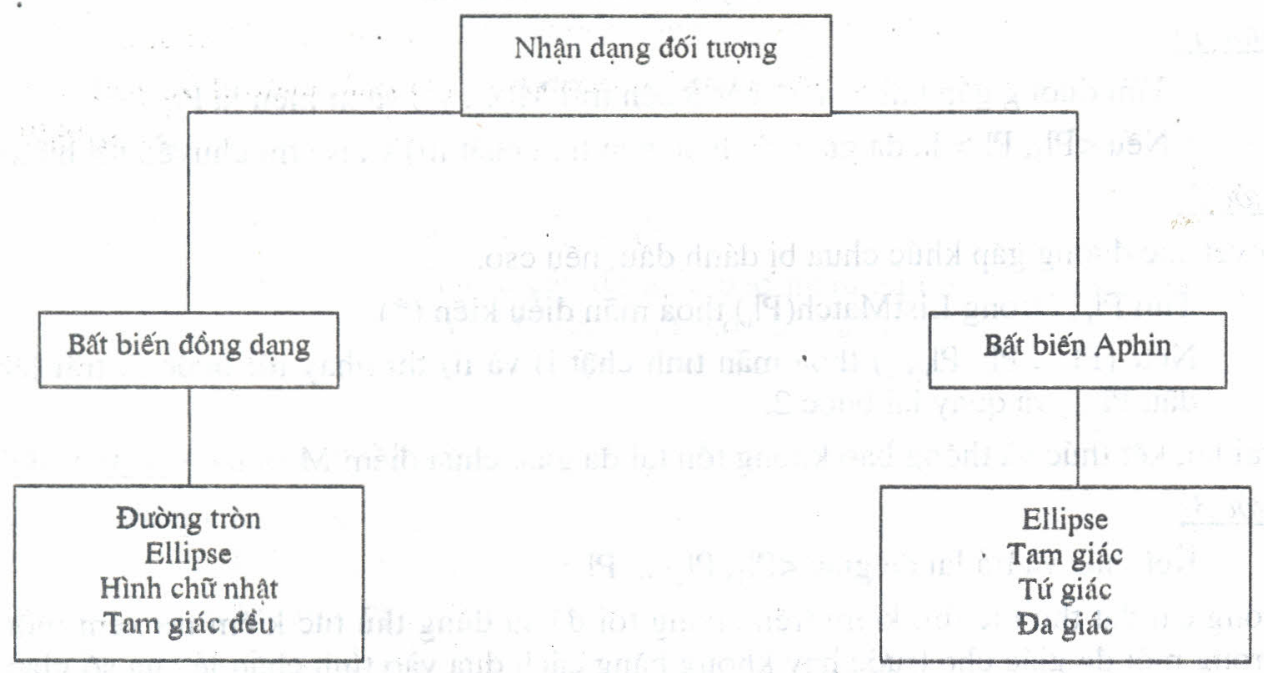

Hinh 2. Sơ đồ phân loại các đôi tượng theo bất biến 


\subsection{Xấp xỉ đa giác theo bất biến đồng dạng}

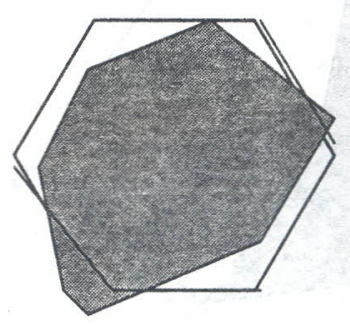

Hình 3: Xấp xỉ đa giác bởi một đa giác mẫu

Theo [5] một đa giác với các đỉnh $\mathrm{V}_{\mathrm{o}}, \ldots, \mathrm{V}_{\mathrm{m}-1}$ được xấp xỉ với đa giác mẫu $\mathrm{U}_{0}, \ldots \mathrm{U}_{\mathrm{n}-1}$ với độ đo xấp xỉ như sau:

$$
E(V, U)=\min _{0 \leq d \leq m-1} \sqrt{\frac{\Delta_{d}}{n}}
$$

Trong đó

$$
\Delta_{\mathrm{d}}=\min _{0 \leq \theta \leq 2 \pi, \alpha \in \mathrm{R}^{2}} \sum_{\mathrm{j}=0}^{\mathrm{n}-1}\left\|\mathrm{kR}_{\theta} \mathrm{U}_{\mathrm{j}}+\mathrm{a}-\mathrm{V}_{(\mathrm{j}+\mathrm{d}) \bmod \mathrm{m}}\right\|^{2}, \mathrm{k}=\sqrt{\frac{\operatorname{area}\left(\mathrm{V}_{0} \ldots \mathrm{V}_{\mathrm{m}-1}\right)}{\operatorname{area}\left(\mathrm{U}_{0} \ldots \mathrm{U}_{\mathrm{n}-1}\right)}} \text {, với } \mathrm{R}_{\theta} \text { là }
$$

phép quay quanh gốc tạo độ một góc $\theta$.

Cũng theo [5] $\Delta_{\mathrm{d}}$ được tính hiệu quả bằng công thức sau:

$$
\Delta_{d}=\sum_{j=0}^{n-1}\left|V_{(j+d) \bmod m}\right|^{2}-\frac{1}{n}\left|\sum_{j=0}^{n-1} V_{(j+d) \bmod m}\right|^{2}+k^{2} \sum_{j=0}^{n-1}\left|U_{j}\right|^{2}-2 k\left|\sum_{j=0}^{n-1} U_{j} \bar{V}_{(j+d) \bmod }\right|
$$

Ở đây $U_{j}, V_{j}$ được hiểu là các số phức tại các đỉnh tương ứng (trong [5] thì $\mathrm{k}=1$ ). Khi $\mathrm{m}$ >n thì độ phức tạp tính toán rất lớn (cách tiếp cận tương tự có thể xem [8])

Với các hình đặc biệt như hình tròn, hình chữ nhật, hình xác định duy nhất bởi tâm và một đỉnh (đa giác đều) ta có thể vận dẹ̣ng các phương pháp đơn giản hơn như bình phương tối thiểu, các bất biến thống kê và hình học.

Đinh nghĩa 5: Cho đa giác $\mathrm{P}_{\mathrm{g}}$ có các đỉnh $\mathrm{U}_{0}, \mathrm{U}_{1}, \ldots \mathrm{U}_{\mathrm{n}}\left(\mathrm{U}_{\mathrm{o}} \equiv \mathrm{U}_{11}\right)$. Khi đó mô men bậc $\mathrm{p}$ + q (xem [6] được xác định như sau)

$$
M_{p q}=\iint_{P g} x^{p} y^{q} d x d y
$$

Trong thực hành để tính tích phân trên người ta thường sử dụng công thức Green hoặc có thể phân tích phần bên trong đa giác thành tổng đại số của các tam giác có hướng $\Delta O U_{i} U_{i+1}$. 


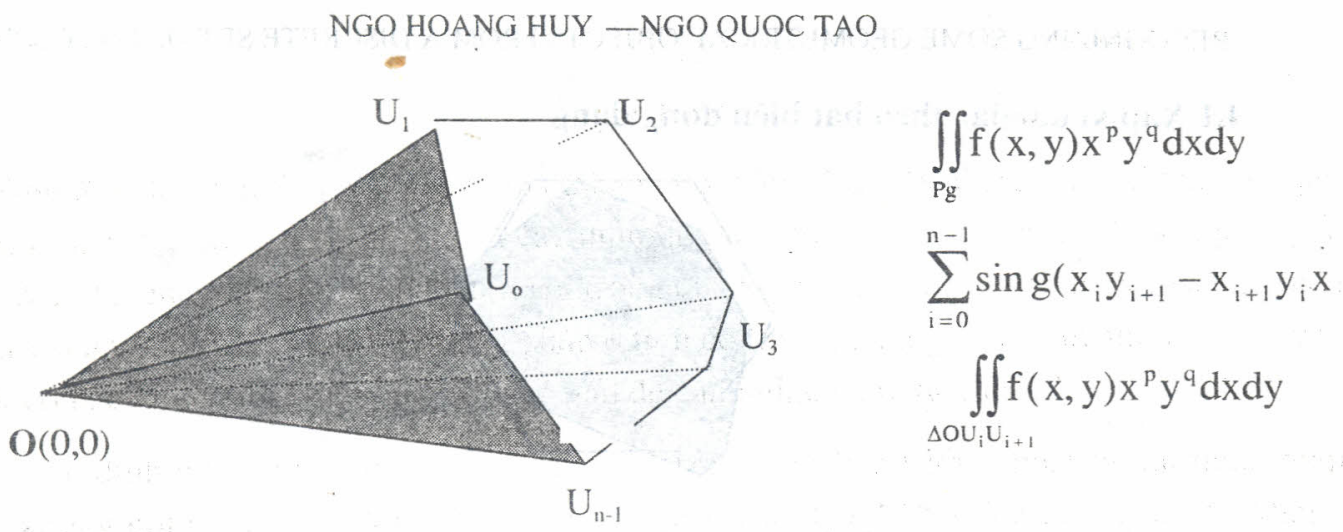

Hình 4. Phân tích miền đa giác thành tổng đại số các miền tam giác

\section{a. Xấp xỉ đa giác bằng đường tròn}

Dìng phương pháp bình phương tối thiểu, ta có độ đo xấp xỉ:

$$
E(P g, C r)=\min _{a, b, c \in R} \sqrt{\frac{1}{n} \sum_{i=1}^{n}\left(x_{i}^{2}+y_{i}^{2}+a x_{i}+b y_{i}+c\right)^{2}}
$$

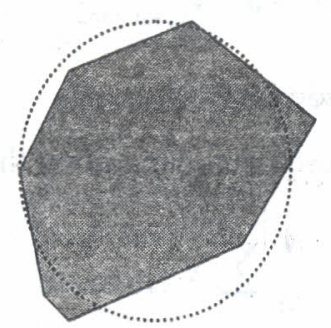

Hình 5. Xấp xỉ đa giác bằng hình tròn.

\section{b. Xấp xi đa giác bằng ellipse.}

Cũng như đối với đường tròn phương trình xấp xỉ với ellipse được cho bởi công thức:

$$
E(P g, E l)=\min _{a, b, c, d, e \in R} \sqrt{\frac{1}{n} \sum_{i=1}^{n}\left(x_{i}^{2}+a y_{i}^{2}+b x_{i} y_{i}+d y_{i}+e\right)^{2}}
$$

Một biến thể khác của phương pháp bình phương tối thiểu khi xấp xỉ các đường cong bậc hai được đưa ra trong [7].

\section{c. Xấp xỉ đa giác bỏi hinh chũ nhât.}

Sử dụng tính chất diện tích bất biến qua phép quay, xấp xỉ theo diện tích như sau:

Gọi $\mu_{11}, \mu_{20}, \mu_{02}$ là các mô men bậc hai của đa giac (tính theo diện tích). Khi đó góc quay được tính bởi công thức sauA:

$$
\operatorname{tg} 2 \varphi=\frac{2 \mu_{11}}{\mu_{20}-\mu_{02}}
$$

Gọi diện tích hình chữ nhật nhỏ nhất có các cạnh song song với các trục quán tính và bao quanh đa giác $\mathrm{Pg}$ là $\mathrm{S}$.

Kí hiệu $\mathrm{E}(\mathrm{Pg}, \operatorname{Rect})=\sqrt{\mathrm{S}-\operatorname{area}(\mathrm{Pg})}$ 


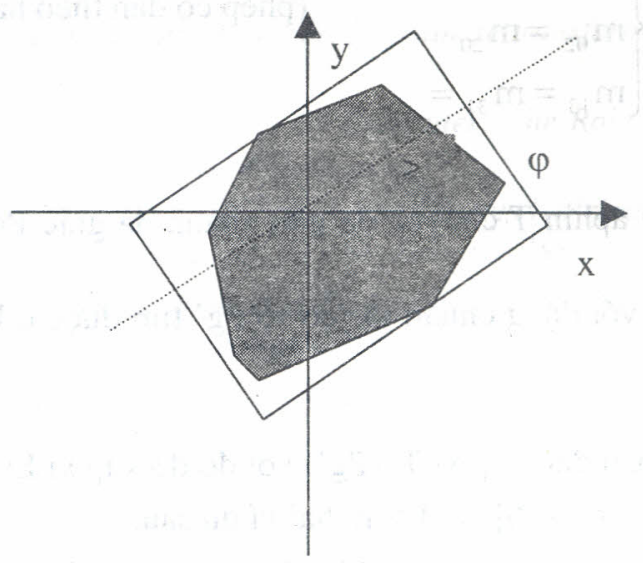

Hình 7. Xấp xỉ đa giác bằng hình chữ nhật

\section{d. Xấp xỉ đa giác bỏi đa giác đều n canh.}

Gọi $\mathrm{M}\left(\mathrm{x}_{0}, \mathrm{y}_{\mathrm{o}}\right)$ là trọng tâm của đa giác, lấy một đỉnh $\mathrm{Q}$ tùy ý của đa giác, xét đa giác đều $\mathrm{n}$ cạnh $\mathrm{Pg}$ 'tạo bởi đỉnh $\mathrm{Q}$ với tâm là $\mathrm{M}$.

Kí hiệu $\mathrm{E}\left(\mathrm{Pg}, \mathrm{Pg}^{\prime}\right)=\sqrt{\left|\operatorname{area}(\mathrm{Pg})-\operatorname{area}\left(\mathrm{Pg}^{\prime}\right)\right|}$

$\mathrm{E}\left(\mathrm{Pg}, \mathrm{E}_{\mathrm{n}}\right)=\min \mathrm{E}\left(\mathrm{Pg}, \mathrm{Pg}{ }^{\prime}\right)$ khi $\mathrm{Q}$ chạy khắp các đỉnh của đa giác.

\subsection{Xấp xỉ đa giác theo bất biến aphin}

Trong [6] đưa ra mô hình chuẩn tắc về bất biến aphin, cho phép chúng ta có thể chuyển bài toán xấp xỉ đối tượng bởi bất biến aphin về bài toán xấp xî̉ mẫu trên các dạng chuẩn tắc. Như vây có thể dựa vào việc đối sánh các đối tượng với các mẫu bởi các bất biến đồng dạng, chẳng hạ việc xấp xỉ bởi tam giác, hình bình hành, ellipse tương đương với xấp xỉ tam giác đều, hình vuông, hình tròn v. v... Thủ tục xấp xỉ theo bất biến aphin một đa giác với hình cơ sở được thực hiện tuần tự như sau:

\section{Bước 0:}

Phân loại bất biến aphin các dạng hình cơ sở

\begin{tabular}{|c|c|c|}
\hline sở & Dạng hình cơ & Dạng chuẩn tắc \\
\hline & Tam giác & Tam giác đều \\
\hline hành & Hình bình & Hình vuông \\
\hline & Ellipse & Đường tròn \\
\hline & $\ldots$. & .... \\
\hline
\end{tabular}

Bước 1:

Tìm dạng chuẩn tăc cơ sỏ Pg' thoả mãn điều kiện: 


$$
\begin{gathered}
\text { NGO HOANG HUY - NGO QUOC TAO } \\
\begin{cases}\mathrm{m}_{01}=\mathrm{m}_{10}= & \text { (phép tịnh tiến) } \\
\mathrm{m}_{02}=\mathrm{m}_{20} & \text { (phép co dãn theo hai trục } \mathrm{x}, \mathrm{y}) \\
\mathrm{m}_{13}=\mathrm{m}_{31}= & \end{cases}
\end{gathered}
$$

Buóó 2:

Xác định biến đổi aphin $\mathrm{T}$ chuyển đa giác thành đa giác $\mathrm{Pg}$ ở dạng chuẩn tắc (thoả mãn tính chất $(* *))$.

Xấp xỉ đa giác $\mathrm{Pg}$ với dạng chuẩn tắc cơ sở $\mathrm{Pg}$ ' tìm được ở bước 1 với độ đo xấp xỉ $\mathrm{E}(\mathrm{Pg}$, Pg').

\section{Buóóc 3:}

Kết luận, đa giác ban đầu xấp xỉ $\mathrm{T}^{-1}\left(\mathrm{Pg}^{\prime}\right)$ với độ đo xấp xỉ $\mathrm{E}(\mathrm{Pg}, \mathrm{Pg}$ ')

Đối với các bước 1 trong [6] đã đưa ra hai ví dụ sau:

Vi clụ 1: Tôn tại duy nhất tam giác ctều $\Delta P_{1} P_{2} P_{\text {: }}$ thoú mãn tính chất (**w) là

$$
\mathrm{P} 1=(0,-2 \alpha), \mathrm{P} 2=(\sqrt{3} \alpha, \alpha), \mathrm{P} 3=(-\sqrt{3} \alpha, \alpha), \alpha=\frac{\sqrt[4]{2} \sqrt[8]{3}}{\sqrt{3}}
$$

Vi dụ 2: Tôn tụi hinh vuông $P_{1} P_{2} P_{3} P_{4}$ thoả mãn tinh chất (**)

* Hình vuông thứ nhất có 4 đỉnh tương ứng là $(-p,-p),(-p, p),(p,-p),(p, p)$,

với $\mathrm{p}=\sqrt[4]{\frac{3}{4}}$

* Hình vuông thứ hai có 4 đỉnh tương ứng là $(-p, 0),(p, 0),(0,-p),(0, p)$, với $p=\sqrt[4]{3}$

\section{KẾT LUẠN.}

Bài báo này đã đưa ra phương pháp trích chọn đa giác dựa vào điểm gieo và một số kiểu xấp xỉ đa giác này bởi các hình cơ sở. Bài báo này còn đề cập đến hai cách tiếp cận bất biến đồng dạng và bất biến aphin. Từ đó chúng tôi đưa ra cách xấp xỉ mẫu một đa giác thành một hình cơ sở. Cách tiếp cận này cho phép nhận dạng được các đối tượng phẳng sau quá trình vectơ hoá.

\section{LÒ̀I CÁM ONN.}

Chúng tôi xin trân thành cảm ơn GS TS Bạch Hưng Khang đã tạo điều kiện cho chúng tôi nghiên cứu. Chúng tôi cảm ơn PTS Lương Chi Mai đã đóng góp những ý kiến qúy báu trong quá trình hình thành bài báo này.

\section{TÀI LIÊU THAM KHẢO.}

1. Bach Hung Khang, Luong Chi Mai, Ngo Quoc Tao, et, al., An Examination of Techniques for Raster - to - Vector Process and Implementation of Software Package for Automatic Map Data EntryMapscan, Journal of Computer Science and Cybernetics vol 12. No 2, 1996, pp. 21 - 29.

2. Ralf Harmut Guting and Markus Schneider. Realm: A foundation for Spatial Data Types in Datahase System.s. In David Ahel and Beng Chin Ooi. Advance in Spatial Datahase - Third International Symprosium, SSD 93 Singapore, June 23 - 25, 1993 Proceeding, Berlin, June 1993, Springer - Verlag. 
3. Ngo Quoc Tao. A Formal Specification of Realm - Spatial Data Types. UNU/IIST Technical Report 71. April 1996.

4. Ngo Quoc Tao, Extracting Invariants Based on Coordinate transformations, Tap chí Tin họ và Điều khiến học tập IX số 4, 1993, pp. 27 - 32.

5. W. Eric, L. Grimson, Ohject Recognition By Computer: The Role of Geometric Constraints, The MIT Press, pp. 207 - 209, London, England, 1990.

6. K. Voss and H. Sueses, Invariant Fitting of Planar Ohjects hyPrimitives. IEEE Trans, Pattern Analysis and Macine Intelligence, Vol, 19, No 1, pp. 80 - 84, January, 1997.

7. M. Werman, Z, Geyzel, Fitting A Second Degree Curve In The Presence Of Error, IEEE Trans. Pattern Analysis and Machine Intelligence, Vol. 17, No 2, pp 207 - 211, 1995.

9. R. Mehrota, J. E. Gary, Similar - Shape Retrieval In Shape Data Management, IEEE Computer Society, pp. 57 - 62, September 1995. 